\title{
Über ein Verfahren zur Wertbestimmung des Safrans.
}

\author{
Von
}

\author{
Dr. B. Pfyl in Berlin und Dr. W. Scheitz in Meerane.
}

Das vorzuschlagende Verfahren soll, wie schon die Überschrift andeutet, nicht bloß dazu dienen, um einen im Sinne der "Vereinbarungen" unverfälschten Safran mit analytischen Zahlen zu bewerten, sondern auch dazu, um in den üblichen Safranverfälschungen die Menge des reinen $d . h$. von Griffeln freien Safrans quantitativ festzulegen, Für den ersten Fall sind bereits Verfahren in Vorschlag gebracht worden. Für den zweiten Fall, wobei es sich hauptsächlich um gepulverten Safran, worin fremde Bestandteile kaum mikroskopisch zu erkennen sind, oder um extrahierten und gepulverten Safran handelt, findet sich bisher kein Wegweiser.

Die bekanntesten der erwähnten Vorschläge beruhen auf colorimetrischer Grundlage, indem der Safran nach der Menge des in ihm enthaltenen Farbstoffes auf Grund einer Vergleichslösung nach den bekannten colorimetrischen Prinzipien abgeschätzt wird. Vin ass a ${ }^{1}$ ) der zuerst als Vergleichslösung eine $10^{0} / 0$-ige Kaliumbichromatlösung vorschlug, gibt selbst an, daß bei einer Beurteilung des Safrans nach diesem Verfahren sehr vorsichtig vorgegangen werden müsse. Dow zard ${ }^{2}$ ) hat die Kaliumbichromatlösung durch eine Chromsäurelösung ersetzt. Über dieses Verfahren hat sich Ka y s e ${ }^{3}$ ) in einer Generalversammlung des Verbandes selbständiger öffentlicher Chemiker geäußert. Hiernach hat ein und derselbe Analytiker bei Anwendung des Verfahrens von Dow z a r d bei demselben Safran Zahlen gefunden, die gegen $40^{\circ} \%$ von einander abweichen. Kayser bezeichnet daher mit Recht das Verfahren als unbrauchbar. Jonscher ${ }^{4}$ ) hat damit ebenfalls seblechte Erfahrungen gemacht; er benutzt als Vergleichsflüssigkeit eine Safranlösung, wodureh es angeblich möglich wird, wenigstens die Volloder Minderwertigkeit eines Safrans zahlengemäB auszudrücken. Kayser gibt zu, daß dadurch wesentliche Fehlerquellen ausgeschaltet werden, daß jedoch solche bestehen bleiben, die teils subjektiver Art sind, teils darin liegen, daß die gelbe Farbe an sich sehr ungeeignet für colorimetrische Bestimmungen ist.

Einen von colorimetrischen Vorfahren verschiedenen Weg haben nur Hilger und $\mathrm{Kuntze}^{5}$ ) eingeschlagen. Ihr Vorschlag besteht darin, daß man das im Safran enthaltene Crocetin mittels Normal-Salzsäure im Wasserdampfstrom abscheidet, auf gewogenem Filter sammelt und nach dem Auswaschen zur Wägung bringt. Sie erhielten zwar noch sehr auseinandergehende Ergebnisse, stellten jedoch die Prognose, daß bei weiterer Ausarbeitung der quantitativen Abscheidung von Crocetin brauchbare Ergebnisse zur Wertbestimmung des Safrans zu erhalten seien. Der eine ${ }^{6}$ ) von uns hatte sich alle Mühe gegeben, um aus dem Verfahren etwas zu machen, indem er einerseits den Einflul der verschiedenen Säuren von verschiedener Konzentration auf die Crocetinfällungen studierte, andererseits versuchte, das abgeschiedene Crocetin oxydimetrisch

1) Arch. d. Pharm. 1892, 280, 353.

2) Pharm. Journ. 1898, 61, 443. (Liverpool Chemists Assoc.)

3) Vergl, Zeitschr, öffentl. Chem. 1907, 13, 423-424.

ક) Zeitsehr. offentl. Chem. 1905, 11, 444 .

5) Arch. Hyg. 1888, 8, 468.

6) Scheitz, Inaugural-Dissertation. München 1906. 
mit Permanganat zu bestimmen. Aber er kam selbst bei einem und demselben Safran nie zu einigermafen konstanten Werten. Wie sich bei späteren Versuchen zeigte, geben übrigens auch andere Pflanzenfarbstoffe, namentlich der von Carthamus tinctorius, der vielfach als Verfälschungsmittel angewendet wird, ähnliche Niederschläge. Das Verfahren hätte daher schon aus diesem Grunde einen nur beschränkten Wert gehabt.

Nach den Arbeiten von Hilger und Kuntze'), Hilger und Schüler ${ }^{2}$ ), Kayser $\mathbf{r}^{3}$ ) lag es sehr nahe, die Menge des im Safran enthaltenen Zuckers als Maßstab für die Güte der unverfälschten Droge und gleichzeitig als Grundlage für die quantitative Bestimmung des Rein-Safrans in verfälschtem Safran heranzuziehen. Da es uns im Verlaufe der bereits mitgeteilten Versuche ${ }^{4}$ ) gelungen war, einen wesentlich besseren Einblick in die Verhältnisse zu bekommen, in denen die im Safran enthaltenen Zucker vorliegen, so konnten diese Erfahrungen verwertet werden.

Es scheinen uns hierzu hauptsächlich jene Zuckerarten (einschließlich Glykoside) geeignet, die wir im Chloroformextrakt feststellten, teilweise krystallinisch abschieden und als Glykoside erkannten. Da die Zucker des Handels nicht in Chloroform übergehen, so können sie daher niemals als Ersatz dieser Substanzen gebraucht werden. Da wir ferner auch qualitativ feststellten, daß weder die Griffel noch die üblichen Verfälchungsmittel des Safrans einen Stoff enthalten, der in Chloroform losslich ist und nach der Einwirkung von Säuren Fehling'sche Lösung reduziert, so haben wir uns entschlossen, die dem Zucker des Chloroformextraktes entsprechende Menge des reduzierten Kupfers als Maßstab zur Bestimmung des Rein-Safrans heranzuziehen.

Eine Reihe von Versuchen führte schlieblich zur Festlegung des nachfolgenden Arbeitsverfahrens, welches bei reinem Safran übereinstimmende Werte lieferte:

Der Safran wird im Wassertrockenschranke sehr scharf getrocknet, dann fein zerrieben und nochmals getrocknet. Davon wiegt man $5 \mathrm{~g}$ auf einer feinen Handwage ab und behandelt sie im Soxhlet'schen Extraktionsapparate 1 Stunde lang mit Petroläther, um das störende Fett zu entfernen. Es wird sodann der der Papierpatrone anhaftende Petroläther durch vorsichtiges Erwärmen im Wassertrockenschrank vollständig verjagt und dieselbe Substanz ebenfalls im Soxhlet'schen Fxtraktionsapparate 2 Stunden lang mit Chloroform ausgezogen. Nach dem Abdestillieren des Lösungsmittels verbleibt ein brauner Rückstand, der mit heibem Aceton aufgenommen wird. Diese Lösung spült man sorgfältig in ein kleines Becherglas; das etwas mehr als $25 \mathrm{ccm}$ Wasser enthält. Das Volumen von $25 \mathrm{~cm}$ ist mit einem Teilstrich am Becherglas markiert. Es wird nun das Becherglas mit einem durchlochten Uhrglase bedeckt und das Aceton über einer kleinen Flamme auf dem Drahtnetz völlig weggekocht. Dann gibt man $5 \mathrm{~cm}$ Normal-Salzsäure hinzu und hält die Flüssigkeit etwa 15 Minuten unter teilweisem Ersatz des verdampften Wassers bis zur Marke im Kochen. Es tritt hierbei unter Auftreten des Geruches nach Safranöl und Abscheidung brauner schmieriger Massen die Spaltung des Glykosids ein. Nach dem Erkalten läßt sich die Flüssigkeit leicht filtrieren, wobei man das Filter mit wenig Wasser nachwäscht. Das schwach gelb gefärbte Filtrat wird nun nach der Neutra-

1) Arch. Hygiene 1888, 8, 468 .

2) Schüler, Inaugural-Dissertation. München 1899.

3) Ber. Deutsch. Chem. Gesellsch. 1884, 17, 2228.

4) Diese Zeitschrift 1908, 16, 237. 
lisation mit Normal-Alkalilauge zu einer Allihn'schen Zuckerbestimmung benutzt. Man kocht eine Mischung von je $25 \mathrm{ccm}$ der getrennten Fehling'schen Lösungen mit $50 \mathrm{cem}$ Wasser einmal auf, versetzt sodann mit der zu prüfenden Flüssigkeit und hält vom Wiederbeginn des Kochens an genau 2 Minuten im Sieden. Das abgeschiedene Kupferoxydul wird dann in der bekannten Weise im Asbestfiltrierrohre abgesaugt, ausgewaschen, mit Alkohol und Äther nachgespült und im Wasserstoffstrome zu metallischem Kupfer reduziert und als solches gewogen. Es ist durchaus notwendig, nur sehr gut getrockneten Safran zu verwenden und auch bei der Operation selbst darauf zu achten, daß keine Spur Wasser in den Extraktionsapparat gelangt, da sonst der Wert für metallisches Kupfer leicht zu hoch gefunden wird. Auch hat man sich vor der Extraktion mit Chloroform davon zu überzeugen, dab der dem Pflanzenpulver anhaftende Petroläther vollständig verjagt ist, da andernfalls das Chloroform nur unvollkommen einwirkt und dann zu niedrige Werte gefunden werden.

Trotz der bereits mitgeteilten qualitativen Ergebnisse, wonach der Chloroformextrakt der Griffel und der üblichen Verfälschungsmittel des Safrans nach der Einwirkung von Säuren nicht reduziert, haben wir der Kontrolle und Sicherheit halber die üblichen Verfälschungsmittel genau nach dieser Methode untersucht. Die hierbei gemachten Beobachtungen seien hier kurz mitgeteilt:

1. Zunächst wurde ein Safran von bekannter Kupferzahl nach dem Entfernen der Narben nochmals analysiert. Man beobachtete, daß das für den ursprünglichen Safran gefundene Kupfer von 0,1988 auf $0,04 \mathrm{~g}$ des minderwertigen Rückstandes zurückging. Sorgfältig von den Narben abgetrennte Griffel gaben an Chloroform keinerlei reduzierende Substanzen ab.

2. Lignum Santali. Der stark grün fluoreszierende Chloroformextrakt hinterläßt einen erheblichen Rückstand. Nach der Spaltung und Neutralisation scheidet sich ein reichlicher Niederschlag $a b$, von dem abfiltriert werden muß. Die klare Lösung reduziert Fehling'sche Lösung nur äuBerst schwach, sodab der entstandene Niederschlag von Kupferoxydul quantitativ nicht in Betracht kommt.

3. Rhizoma Curcumae. Der ebenfalls grün fluoreszierende Chloroformauszug reduziert nach dem Entfernen des Chloroforms Fehling'sche Lösung stark. Nach der Spaltung und Neutralisation jedoch bewirkt die von den ausgeschiedenen Stoffen abfiltrierte Flüssigkeit nur noch eine äußerst geringe kaum wägbare Kupferabscheidung.

4. Lignum Campechianum gibt wenig Substanz an Chloroform ab. Nach der Spaltung wird Fehling'sche Lösung nicht reduziert.

5. Flores Rhoejodos geben wenig Substanz an Chloroform ab. Der Rückstand reduziert nach der Einwirkung von Säuren nur äußerst gering.

6. Flores Paeoniae verhalten sich ebenso.

7. Honig, der zur. Unterbringung anorganischer Beschwerungen bekanntlich häufig dem Safran beigemengt wird, gibt an Chloroform keinen Stoff ab, der F ehling'sche Lösung reduziert.

8. Ringelblume (Calendula officinalis L.) reduziert vor und nach der Einwirkung von Säuren nicht.

9. Safflor (Carthamus tinctorius) verhält sich ebenso.

Der sogenannte Cap-Safran sowie die spanische Golddistel, die ehenfalls als Verfälschungsmittel verwendet werden sollen, waren trotz zahlreicher Bemühungen einiger 
erster Drogenhäuser nicht aufzubringen. Sie haben also wohl nur sehr untergeordnete Bedeutung.

Es ist somit festgestellt, dab in einem griffelhaltigen oder in verfälschtem Safran im wesentlichen nur die Narben des Safrans an Chlorofom lösliche Substanzen abgeben, die nach der Einwirkung von Säuren Kupferlösung in mebbarer Meng"e reduzieren.

Im Hinblick auf dieses erfreuliche Ergebnis haben wir eine Anzahl der üblichsten Handelssorten des Safrans untersucht, um festzulegen, ob der Gehalt an den in Frage kommenden reduzierenden Stoffen bei Safransorten verschiedener Herkunft weit auseinandergeht. Als die für Deutschland in Betracht kommenden Safransorten des Handels werden in den "Vereinbarungen" bezeichnet:

1. Osterreichischer Safran (selten),

2. Französischer Safran,

3. Spanischer Safran.

Trotz aller Bemühungen einiger erster Drogenhäuser war österreichischer Safran nicht zu beschaffen. Er scheint demnach vollständig vom Markte verschwunden zu sein.

Die Versuchsergebnisse bei den übrigen Safransorten sind in der folgenden Tabelle zusammengestellt:

\begin{tabular}{|c|c|c|c|c|}
\hline No. & Handelsmarke & $\begin{array}{l}\text { Preis für } \\
100 \mathrm{~g} \text { in } \\
\text { Mark }\end{array}$ & Aussehen & $\begin{array}{c}\text { Gefundenes } \\
\text { Kupfer } \\
g\end{array}$ \\
\hline 1 & Crocus Gatinais electus. & $12,-$ & Dunkelrot, nur reine Narben. . & 0,2090 \\
\hline 2 & , . . . . . & $12,-$ & $" \quad " \quad n \quad n .$. & 0,1996 \\
\hline 3 & $\pi \quad . . .$. & $12,-$ & , & 0,2010 \\
\hline 4 & Spanisch. Safran eleg. Ia & $11,-$ & $n \quad n \quad n$ & 0,1756 \\
\hline 5 & " natur. sup. . & 9,50 & Ansätze von Griffeln . . . . . . & 0,1710 \\
\hline 6 & Crocus Hispanicus. . . & 9,50 & nur sehr geringe Ansätze von Griffeln & 0,1670 \\
\hline 7 & $\pi \quad, \quad I a$. & $9,-$ & Ansätze von Griffeln . . . . & 0,1576 \\
\hline 8 & "No. 2. & 8,50 & $\Rightarrow \quad \# \quad * \quad . \quad .$. & 0,1610 \\
\hline 9 & $" \quad$ No. 3. & $8,-$ & reichlich Griffel enthaltend . . & 0,0930 \\
\hline 10 & Marke ${ } \mathrm{El}^{\prime} "$. . . & 8,50 & dunkelrot, wenig Griffel . & 0,1370 \\
\hline 11 & "G. . . . & $8,-$ & $\Rightarrow \quad n \quad n \cdot$. & 0,1230 \\
\hline 12 & D. . & 6,25 & viele Griffel enthaltend . & 0,0780 \\
\hline 13 & n $\quad \mathbf{E} . \ldots$. & $6,-$ & $\pi \quad \% \quad \% \quad .$. & 0,0640 \\
\hline 14 & Französischer Safran, eleg. & $12,-$ & hellrot, nur reine Narben . . . & 0,1500 \\
\hline 15 & $" \quad$, natur. . . & 9,50 & mit geringen Ansätzen von Griffeln & 0,1502 \\
\hline 16 & $n \quad, \quad n \quad . \quad$. & $9,-$ & \# $\quad$ n $\quad$ n. & 0,1476 \\
\hline 17 & Span. Safran, gemahlen I a & 10,50 & ziegelrotes Pulver . . & 0,1356 \\
\hline 18 & " $\quad$ gemahlen II a & $10,-$ & n $\quad$. . . . & 0,1192 \\
\hline 19 & Safran des Kleinhandels & $2,-$ & braunrotes Pulver . . . . . . & 0,0474 \\
\hline
\end{tabular}

Auf. Grund der angeführten Zahlen hat man zweifellos schon Anhaltspunkte um die Minder- oder Vollwertigkeit eines Safrans zu erkennen. Aber man ist noch nicht imstande, die Menge des reinen Safrans in einem griffelhaltigen oder verfälschtem Safran quantitativ, d. h. in Prozenten auszudrücken. Man würde z. B. zu falschen 
Ergebnissen gelangen, wenn man bei einem verfälschten Safran, der die Kupferzahl 0,0661 gibt, etwa nach dem Ansatz:

$$
0,2090 \mathrm{Cu}: 100 \%=0,0661 \mathrm{Cu}: \times{ }^{0} / 0 ;
$$

$\mathrm{x}=31,6 \%$ reinen Crocus Gatinais electus berechnete.

Denn bekanntermaßen geht die Abscheidung des Kupferoxyduls aus Fehling'scher Lösung nicht proportional der vorhandenen Zuckermenge vor sich; sie ist vielmehr mit der Konzentration der Zuckerlösung variabel. Man muß daher zuvor verschiedene Mengen ein und desselben Safrans analysieren, damit die $Z$ wischenwerte mit annähernder Genauigkeit durch Interpolation gefunden werden können. Als Grundlage wurde die beste Sorte Crocus Gatinais electus gewählt; die Untersuchung ergab:

$\begin{array}{lccccccccc}\text { Angew. Subst. } & .5,0 & 4,5 & 4,0 & 3,5 & 3,0 & 2,5 & 2,1 & 1,2 & 1,0 \mathrm{~g} \\ \text { Kupfer . . . } & 0,2090 & 0,1870 & 0,1619 & 0,1120 & 0,0828 & 0,0614 & 0,0476 & 0,0264 & 0,0230 \mathrm{~g}\end{array}$

Vorausgesetzt nun, daß es keinen wesentlich besseren als den von uns zugrunde gelegten Safran gibt und daß man diesen besten als Maßstab für die Wertbestimmung heranzieht, sind hiermit alle Werte für die quantitative Feststellung des reinen Safrans in Gemischen mit Griffeln oder den üblichen Verfälschungsmitteln gegeben.

Die Berechnung wird am zweckmäßigsten ein Beispiel erläutern:

Angewandte Substanz: 5,0 g; gefundenes Kupfer; 0,0661 g; diese Zahl ist in der Tabelle nicht angegeben. 'Wir suchen die nächst niedrigere Zahl; das ist 0,0614 . Diese würde $2,5 \mathrm{~g}$ Safran entsprechen; die nächst höhere aber $3 \mathrm{~g}$. Um die unserer Kupfermenge entsprechende Safranmenge zu finden, müssen wir daher interpolieren. Wir haben dann den Ansatz ${ }^{1}$ ):

$$
\begin{aligned}
& 0,0214: 0,5=0,0047: x \\
& x=0,10 .
\end{aligned}
$$

Diese Differenzzahl muf daher noch den $2,5 \mathrm{~g}$ hinzugezählt werden, um die entsprechende Safranmenge in $5 \mathrm{~g}$ angewandter Substanz zu finden. In $5 \mathrm{~g}$ Substanz sind demnach $2,6 \mathrm{~g}$ oder $52 \%$ echten Safrans enthalten.

Dieses Beispiel war eine Analyse, die der eine von uns ausführte, ohne den geringsten Anhaltspunkt darüber zu haben, wieviel Safran in der ihm tubergebenen Mischung vorlag. Es war tatsächlich eine Mischung von 50\% Safran mit 50\% Carthamus tinctorius. Desgleichen wurden ihm noch zwei unbekannte Mischungen übergeben, deren Untersuchungsergebnisse und deren tatsächlicher Gehalt an Safran hier noch angeführt werden sollen:

I. Angewandte Substanz: $5 \mathrm{~g}$; gefundenes Kupfer: 0,0443 g.

$$
0,0212: 0.9=0,0179: x \quad x=0,8
$$

Es wurden demnach gefunden in $5 \mathrm{~g}$ Substanz $1,2+0,8=2 \mathrm{~g}$ oder $40 \%$ Rein-Safran tatsächlich vorhanden waren $41 \%$.

II. Angewandte Substanz: $5 \mathrm{~g}$; gefundenes Kupfer: $0,0870 \mathrm{~g}$

$$
0,0212: 0,9=0,0106: x \quad x=0,45
$$

Es wurden demnach gefunden in 5 g Substanz $1,2+0,45=1,65$ g oder $33 \%$ ReinSafran, die auch tatsächlich vorhanden waren.

Zum Schlusse ist noch die Frage zu 'erörtern, ob aus den gefundenen Zablen des Reinsafrans zugleich die Menge der tatsächlichen Verfälschung berechnet und angegeben werden kann. Dies wäre zweifellos zu bejahen, wenn es sich immer

1) In diesem Ansatz ist 0,0214 die Differenz zwischen den KupferzahJen, die 3,0 und 2,5 $\mathrm{g}$ Safran entsprechen und 0,0047 die Differenz zwischen der gefundenen und der nächst niedrigeren Kupferzahl. 
um Fälschungen von Crocus Gatinais electus oder der feinsten Sorten handeln würde. Der Fälscher aber wird sich hüten, von so ausgezeichneter Ware auszugehen. Wenn er aber nun z. B. einen spanischen Safran nat, mit 10\% Griffeln verwendet, der nach den Vorschlägen der Freien Vereinigung Deutscher Nahrungsmittelchemiker noch nicht als verfälscht angesehen werden soll, so könnte unsere Berechnung mit der tatsächlich vom Fälscher ausgeführten Mischung natürlich in keiner Weise übereinstimmen.

Man wird sich daher auf Grund der Analysen begnügen müssen anzugeben:

Die fragliche Probe enthält in 100 Teilen rund soviel Gewichtsteile reinen Safran, wie $x$ Gewichtsteilen feinstem Safran von der Kupferzahl 0,209 $\mathrm{g}$ entsprechen.

\section{Zusammenfassung der Ergebnisse.}

1. Der Chloroformextraktrückstand der scharf getrockneten und mit Petroläther behandelten Safrannarben, in welchem durch unsere früheren Untersuchungen Fruktose und ein diesen Zucker lieferndes Glykosid nachgewiesen sein dürften, reduziert auch bei Anwendung relativ kleiner Mengen von Substanz (unter 1 g) Fehling'sche Lösung sehr stark, während kleinere Mengen (unter $5 \mathrm{~g}$ ) von Griffeln sowie der üblichen Verfälschungen des Safrans unter denselben Umständen nicht oder in kaum wägbarer Merige reduzieren,

$5 \mathrm{~g}$ feinster Crocus Gatinais electus geben nach dem beschriebenen ArbeitsVerfahren 0,2090, 0,1996, 0,2010 g Kupfer, während französischer Safran im Mittel etwas weniger Kupfer liefert. Die Kupferzahlen von griffelhaltigen Safrazen liegen bedeutend tiefer.

Nach demselben Verfahren liefern $5 \mathrm{~g}$ Griffel und $5 \mathrm{~g}$ der häufigsten Verfälschungsmittel des Safrans: Carthamus tinctorius und Calendula officinalis nicht eine Spur Kupfer. Auch die anderen Verfälschungsmittel reduzieren nicht oder kaum meBbar. Etwas Vorsicht dürfte vielleicht nur bei Curcuma und Sandelholz am Platze sein.

2. Unter Benützung der Interpolationstabelle auf Seite 351 und unter der Voraussetzung, daß man den von uns gewählten besten Crocus Gatinais electus als Maßstab für die Bewertung heranzieht, kann daher voraussichtlich in allen üblichen Verfälschangen des Safrans die Menge des Reinsafrans quantitativ bestimmt werden.

In 3 unbekannten Mischungen von feinstem spanischem Safran mit der Kupferzahl $0,2090 \mathrm{~g}$ mit Carthamus tinctorius wurde die Menge des Reinsafrans einmal auf $2 \%$, einmal auf $1 \%$ und einmal ganz genau gefunden.

In Anbetracht der Wichtigkeit des Gegenstandes wäre eine Nachprüfung des Verfahrens und die Angabe möglichst vieler Kupferzahlen von reinen und verfälschten Safranen zum Ausbau und zur Verfeinerung dieser sehr erwïnscht, insbesondere da Verfasser sich vorderhand mit diesem Arbeitsgebiete leider nicht weiter befassen können.

Wie uns von privater Seite mitgeteilt wird, ist die Methode bereits mit gutem Erfolge in der Praxis angewendet worden und hat sie sich als durchaus brauchbar erwiesen.

Die in vorliegender Arbeit beschriebenen Versuche wurden in den Jahren $1903-1905$ im Laboratorium für angewandte Chemie an der Kgl. Universität in München ausgeführt. 